\title{
Chemoembolisation with lipiodol and doxorubicin: applicability in British patients with hepatocellular carcinoma
}

\author{
S D Ryder, P M Rizzi, E Metivier, J Karani, Roger Williams
}

\begin{abstract}
Chemoembolisation has been extensively used as primary treatment for unresect-

that this treatment offers little benefit in advanced disease.

(Gut 1996; 38: 125-128)
\end{abstract} able hepatocellular carcinoma (HCC). In this unit, 185 patients with a new diagnosis of HCC not amenable to surgery were seen between 1988 and 1991. Intended therapy for these patients was chemoembolisation with doxorubicin (60 $\mathrm{mg} / \mathrm{m}^{2}$ ) and lipiodol, repeated at six week intervals until it was technically no longer possible or until complete tumour response had been obtained. Chemoembolisation was possible in 67 of the 185 $(37 \%)$. Reasons for exclusion were portal vein occlusion $(n=36)$, decompensated cirrhosis $(n=44)$, distant metastases $(n=5)$, diffuse tumour or unsuitable anatomy (tumour or vasculature) $(n=11)$, patient refusal $(n=11)$, and other $(n=11)$. Patients excluded from treatment survived for a median of 10 weeks (range 3 days-19 months). In patients treated, 18 had small $\mathrm{HCC}(<4 \mathrm{~cm})$ and 49 had large or multifocal HCC. Chemoembolisation was carried out a median of two sessions for small and three sessions for large tumours. Ten of 18 patients with small HCC showed a $50 \%$ or greater reduction in tumour size. Five of 49 patients with large or multifocal tumours showed a response to treatment. Median overall survival for treated patients was 36 weeks (range 3 days -4 years). One patient has subsequently undergone liver transplantation with no recurrence and minimal residual disease at transplantation. Two other patients are alive three years after chemoembolisation, one with no evidence of recurrent disease. No patient was thought suitable for surgery after their response to chemoembolisation. Chemotherapy related complications were seen in $22 \%$. Complications were significantly more common in patients with larger tumours and poor liver reserve. Five patients died as a result of chemotherapy related complications. In conclusion, only one third of UK patients with unresectable HCC are treatable by chemoembolisation. Results with small tumours are encouraging, with a high response rate and the possibility of surgical intervention in previously inoperable disease. Large tumours, however, show a poor response and a significant incidence of side effects, suggesting
Keywords: Chemoembolisation, lipiodol, doxorubicin, hepatocellular carcinoma.

Surgery remains the only curative treatment for hepatocellular carcinoma (HCC). In the UK, most patients present with large tumours and underlying cirrhosis and are not treatable by surgical resection or transplantation. ${ }^{1}$ Medical treatment for advanced HCC has generally produced disappointing results but the advent of targeted chemotherapy using lipiodol in combination with hepatic arterial embolisation has generated considerable interest, with uncontrolled series reporting excellent survival ${ }^{23}$ and low complication rates. ${ }^{45}$ There are, however, patients who are not thought suitable for this treatment because of severe underlying hepatic dysfunction or portal vein involvement, ${ }^{6}$ frequent findings in the predominantly unscreened patients presenting de novo with HCC in the UK. This study aimed to analyse the usefulness of chemoembolisation in an unselected cohort of UK patients in whom surgery was not possible.

\section{Patients and methods}

Between January 1988 and December 1991, 195 patients presented to this unit with HCC. Six patients were treated by orthotopic liver transplantation and four by hepatic resection. The remaining 185 patients were not considered suitable for surgery because of either large or multifocal tumours, replicating hepatitis B virus infection, distant spread of disease, or compounding medical problems. All patients were considered as candidates for chemoembolisation as first line medical treatment. Of the 185 patients unsuitable for surgery, five were non-cirrhotic, 23 had Child's A cirrhosis, 72 Child's B cirrhosis, and 85 Child's $C$ cirrhosis at the time of presentation. The aetiology of the underlying liver disease was hepatitis B in 51, hepatitis C in 47, alcohol in 48 , primary biliary cirrhosis in six, haemochromatosis in four, cryptogenic disease in 11 , and other causes in 18. Criteria for exclusion from chemoembolisation treatment were as follows:

- Hepatic dysfunction (bilirubin $>50 \mathrm{mmol} / \mathrm{l}$ and encephalopathy);

- Renal failure (creatinine $>200 \mu \mathrm{mol} / \mathrm{l}$; 
TABLE I Child's grade of liver cirrhosis in patients treated by repeated chemoembolisation or excluded from treatment

\begin{tabular}{lcc}
\hline Child's grade & Treated & Not treated \\
\hline Non-cirrhotic & 4 & 1 \\
Child's A & 10 & 13 \\
Child's B & 37 & 35 \\
Child's C & 16 & 69 \\
Total & 67 & 118 \\
\hline
\end{tabular}

- Chemotherapeutic agent related (severe cardiac disease, thrombocytopenia $(<50000 /$ $10^{9} / 1$ ), active infection);

- Patient refused;

- Procedure related (unsuitable tumour anatomy (diffuse intrahepatic tumour), unsuitable vascular anatomy, portal vein occlusion (main trunk only, segmental portal occlusion accepted), or pulmonary shunting.

Chemoembolisation was carried out according to a standard protocol. Patients were given intravenous hydration, antibiotics, and $\mathrm{H}_{2}$ antagonists routinely before the procedure. The anatomy was delineated at hepatic angiography beforehand. Selective cannulation of the hepatic artery was followed by injection of a freshly prepared suspension of lipiodol and doxorubicin $\left(60 \mathrm{mg} / \mathrm{m}^{2}\right)$ in a final volume of $100 \mathrm{ml}$. After injection of this suspension into the tumour feeding vessel, this vessel was embolized using particulate material.

The response to therapy was determined by serial computed tomography (CT) scans of the liver and is defined as a $50 \%$ reduction in the maximum tumour diameter. Complications relating to the procedure were noted and chemoembolisation was repeated at six week intervals until the patients either died, became unfit for further treatment, or until anatomically unsuitable for treatment.

\section{Results}

Altogether 118 of 185 (65\%) patients were excluded from treatment. The reasons for this were portal vein occlusion $(n=36)$, decompensated cirrhosis $(n=44)$, distant metastases $(n=5)$, diffuse tumour or unsuitable anatomy (tumour or vasculature) $(n=11)$, patient refusal $(n=11)$, cardiac disease $(n=5)$, renal failure $(n=4)$, and haematological $(n=2)$. Of the 118 patients excluded from therapy, 69 had Child's C cirrhosis and 35 Child's B cirrhosis (Table I). Of these patients, 44 received intravenous chemotherapy (doxorubicin or 5 fluorouracil) and 12 had hormonal therapy with tamoxifen. Larger tumours were also less likely to be considered treatable, with only $20 \%$ of patients with tumours $>8 \mathrm{~cm}$ diameter being offered treatment (Table II).

TABLE II Tumour size in relation to treatment with chemoembolisation

\begin{tabular}{llc}
\hline Tumour size & Treated & Not treated \\
\hline$<4 \mathrm{~cm}$ & 18 & 5 \\
$4-8 \mathrm{~cm}$ & 33 & 39 \\
$>8 \mathrm{~cm}$ & 11 & 43 \\
Multifocal & 5 & 31 \\
\hline
\end{tabular}

RESPONSE TO TREATMENT

Chemoembolisation was possible in $67(36 \%)$ of the 185 patients. Chemoembolisation was carried out a median of two sessions for small tumours $(<4 \mathrm{~cm})$ and three sessions for large or multifocal tumours. A total of 202 sessions were undertaken during the three years studied. In patients with small tumours $(n=18)$, eight of 18 showed a partial response and two had complete responses (overall response rate $56 \%$ ). Of the 49 patients with large or multifocal tumours, only one had a complete response and four a partial response to treatment (overall $10 \%$ response rate). Serum $\alpha$ fetoprotein (AFP) values fell in four of six patients with a small $\mathrm{HCC}$ and in nine of 33 with a large HCC who had raised serum concentrations at the time of diagnosis.

If tumour bulk is calculated from the diameter of the liver lesions, there was a close relationship between tumour bulk and the response to treatment (Fig 1). One patient with multifocal HCC but small tumour bulk (two lesions measuring 1.5 and $2.2 \mathrm{~cm}$ ) had a complete response to treatment and has subsequently undergone liver transplantation with no recurrence (27 months later) and had minimal residual disease present in the explanted liver. No other patient was thought suitable for surgery after the response to chemoembolisation.

A significant response to chemoembolisation was usually produced at the first or second session, if no significant tumour shrinkage was seen after two sessions no benefit in terms of further reduction in radiological tumour size was gained thereafter (Table III).

\section{SURVIVAL}

Overall median survival for the 185 patients who were not treated surgically was 14 weeks (Fig 2). For patients excluded from chemoembolisation, median survival was 10 weeks (range 3 days -19 months). Treated patients had a median survival of 36 weeks (range 3 days-4 years) (Fig 2). The cause of death in the 67 treated patients ( 63 deaths with median follow up of 3 years) was liver failure in 24, gastrointestinal haemorrhage in 11 , metastatic disease and/or cachexia in 21 , chemotherapy related in 5 , non-liver related in 2 . Survival in the 15 patients who had a significant

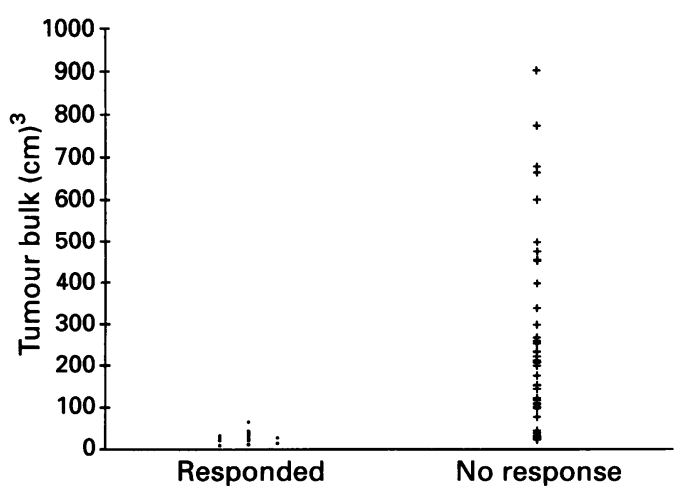

Figure 1: Tumour bulk (calculated from diameter of tumour on imaging) and response to chemoembolisation. 


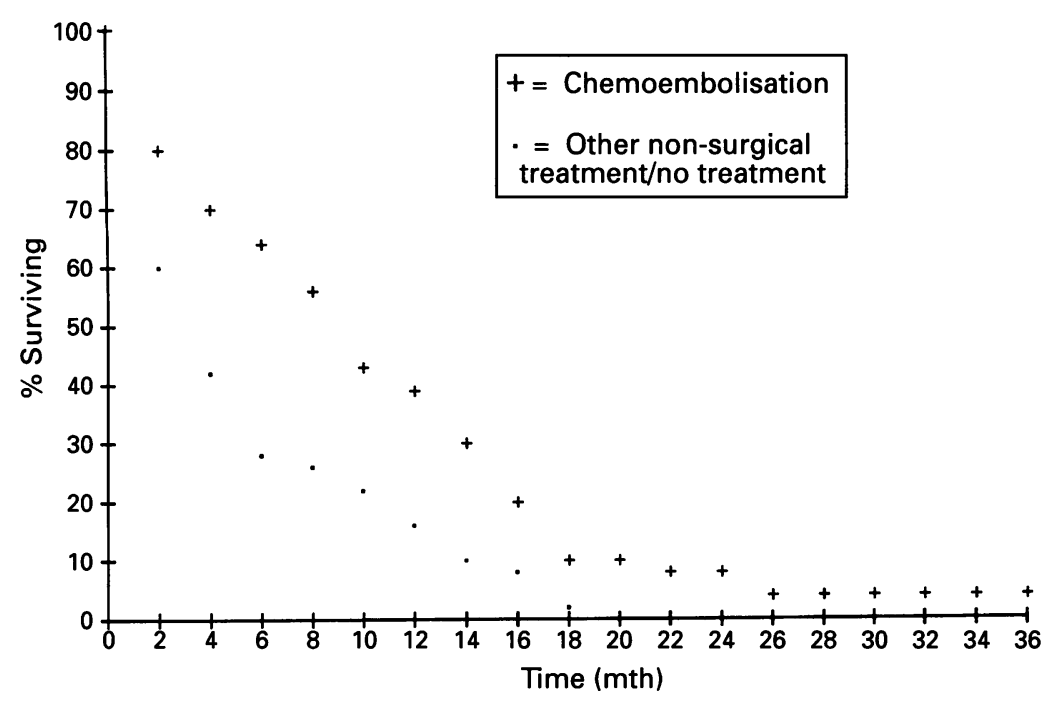

Figure 2: Survival of patients treated by arterial chemoembolisation or other non-surgical treatment (no therapy, intravenous doxorubicin, or tamoxifen).

response to treatment was considerably better than in patients with similar liver impairment (responders: non-cirrhotic $\mathrm{n}=2$, Child's A $n=4$, Child's $B n=8$, Child's C $n=1$; nonresponders: non-cirrhotic $n=2$, Child's $A$ $n=6$, Child's $B n=24$, Child's $C n=15$ ) and similar tumour sizes (mean tumour bulk: responders $34 \mathrm{~cm}^{3}$, non-responders $41 \mathrm{~cm}^{3}$ ) who failed to respond to treatment $(p=0.0002)$ (Fig 3).

Four patients in the treatment group survived for three years or longer after initial treatment. One patient with multifocal disease but small tumour bulk and Child's A cirrhosis (hepatitis B virus related) showed a complete radiological response to a single chemoembolisation and after review showing no regrowth of his HCC six months after therapy underwent orthotopic liver transplantation. Minimal residual tumour was found in one tumour nodule and no viable tumour in the other. $\mathrm{He}$ remains well two years after transplantation with no tumour recurrence. One patient with Child's A cirrhosis and a $2 \mathrm{~cm}$ lesion in the right lobe of the liver had a complete response after two chemoembolisation sessions and remains well three years later with no

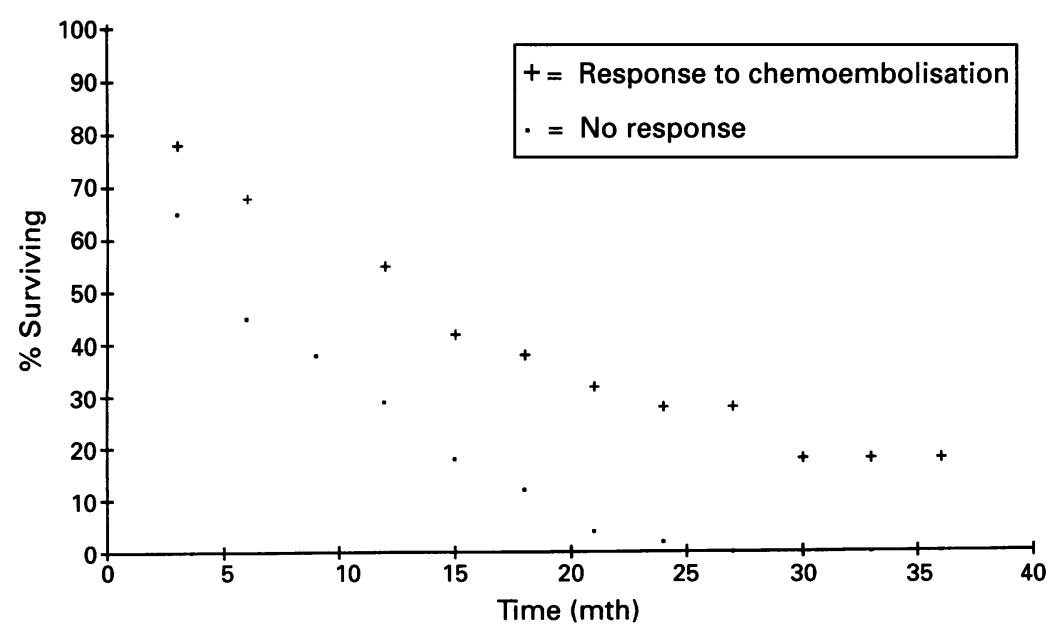

Figure 3: Survival in patients with a significant response to chemoembolisation (50\% or greater decrease in tumour size) and a group of non-responders to treatment matched for age, degree of liver impairment, and tumour size. radiological evidence of HCC. Two patients with a partial tumour response and Child's B cirrhosis had static disease for two years and 18 months respectively. One of these patients is still alive at almost four years from diagnosis, although with slowly progressive disease, and the other, who had radiologically stable disease, died of a myocardial infarction.

\section{TREATMENT COMPLICATIONS}

Initial pain and fever occurred in $90 \%$ of treated patients. Twenty two per cent of treated patients suffered more serious complications of therapy. There were two deaths directly related to the procedure - one patient developed renal failure and one immediate hepatic decompensation. Nine patients developed neutropenic sepsis after chemoembolisation and in three this proved fatal. One patient developed a late bile duct stricture (at four months), which did not seem to be due to tumour invasion and was thought to relate to the chemoembolisation, and one patient bled from a duodenal ulcer. Most of the serious complications occurred in patients with Child's $\mathrm{C}$ cirrhosis and a large tumour bulk (Table IV).

\section{Discussion}

In this study we have shown that chemoembolisation can produce a substantial reduction in the size of the tumour as assessed radiologically but that this beneficial effect is unlikely to be seen in those patients who present with a large tumour bulk or a background of severe liver disease. In addition, a large number of patients are excluded from this treatment. Almost two thirds of the patients were excluded in this series, mainly because of advanced liver disease or portal vein invasion. This large proportion is disappointing, and our finding is supported by the only other series to report exclusion in a chemoembolisation programme, ${ }^{7}$ where $57 \%$ of HCC patients were excluded from treatment. There are considerable differences in the patient population in this series from Africa and the UK group studied here, the main reason for exclusion in the African study was advanced disease but no patient was excluded for portal vein invasion. It is possible that alternative forms of therapy, such as tamoxifen ${ }^{8-10}$ or absolute ethanol injection, ${ }^{11}$ may be possible in these patients but the prognosis at present remains dismal with median survival measured in a few weeks only. Some authors have suggested that portal vein invasion is not necessarily a contraindication to chemoembolisation ${ }^{12}$ providing the

TABLE III Number of chemoembolisation treatments and objective response

\begin{tabular}{lll}
\hline $\begin{array}{l}\text { No of chemoembolisation } \\
\text { sessions }\end{array}$ & $\begin{array}{l}\text { No of } \\
\text { patients }\end{array}$ & $\begin{array}{l}\text { New responses } \\
\text { to therapy }\end{array}$ \\
\hline 1 & 67 & 9 \\
2 & 46 & 6 \\
3 & 38 & 0 \\
4 & 29 & 0 \\
$>5$ & 11 & 0 \\
\hline
\end{tabular}


TABLE IV Complications of chemoembolisation in 67 patients in relation to underlying cirrhosis and tumour size

\begin{tabular}{lcl}
\hline & No treated & No (\%) of complications \\
\hline Child's grade: & & \\
Non-cirrhotic & 4 & $0(0)$ \\
Child's A & 10 & $1(10)$ \\
Child's B & 37 & $9(24)$ \\
Child's C & 16 & $5(31)$ \\
Tumour bulk $\left(\mathrm{cm}^{3}\right):$ & & $1(5)$ \\
$<33$ & 19 & $6(18)$ \\
$33-268$ & 34 & $8(57)$ \\
$>268$ & 14 & \\
\hline
\end{tabular}

embolization is carried out with highly selective cannulation of the feeding vessel. This technique is technically demanding, however, and few centres would consider using it in patients who have main portal trunk involvement.

Our finding that response to chemoembolisation depends on tumour size and underlying liver dysfunction agrees with previous studies. Mondazzi et al, ${ }^{13}$ in a multivariate analysis, showed that age, Child's grade, and tumour size were the most important prognostic factors. The finding of poor response rates in large tumours has not always been the experience of other groups - Ngan et al ${ }^{14}$ reported a very high response rate for chemoembolisation, even in patients with very large $(12 \mathrm{~cm})$ tumours. Ikeda et al ${ }^{15}$ also report a significant response rate in patients with tumours of $8 \mathrm{~cm}$ and above and complete necrosis in a $12 \mathrm{~cm}$ tumour. Both these studies, however, conclude that response rates are better in smaller tumours. Two controlled trials published add further support to our data ${ }^{16} 17$ showing no overall increase in survival in patients with predominantly large tumours and poor underlying liver function. The French experience with large tumours has also not been encouraging; Bismuth et al ${ }^{18}$ found the results in Okuda stage III patients so poor that they abandoned the treatment in this group of patients.

There seems little doubt that some patients with small tumour bulk and good liver function have very good palliation of their tumours using chemoembolisation. Other groups have reported results which compare favourably with historical controls in terms of patient survival $^{19}$ and there is the possibility that tumours previously not thought suitable for surgery or transplantation show a substantial decrease in bulk and become operable. In our series only one patient was thought suitable for transplantation after treatment. The experience in published reports is limited but suggests that chemoembolisation does produce substantial tumour necrosis and may improve the outcome in cases considered to be borderline for resection. ${ }^{20}$ Our current practice is to offer chemoembolisation in combination with hormonal manipulation using tamoxifen wherever this is technically possible. Our results suggest that this is likely to be clinically useful in patients with small tumours and good underlying liver function but is unlikely to be of benefit to patients with tumours above $4 \mathrm{~cm}$ in diameter and carries a much greater risk of complications in this group.

1 Johnson PJ, Williams R. Hepatocellular carcinoma. Postgrad Med F 1979; 23: 100-9.

2 Takayashu K, Shima Y, Muramatsu Y, Moriyama N, Yamada T, Makuuchi M, et al. Hepatocellular carcinoma: treatment with intraarterial iodized oil with and without chemotherapeutic agents. Radiology 1987; 162: 345-51.

3 Kanematsu $T$, Furuta $T$, Takenaka $K$, Matsumata $T$, Yoshida Y, Nishizaki T, et al. A 5 year experience of lipiodolization: selective regional chemotherapy for 200 patients with hepatocellular carcinoma. Hepatology 1989; 10: 98-102.

4 Bronowicki J-P, Vetter D, Dumas F, Boudjema K, Bader R, Weiss A-M, et al. Transcatheter oily chemoembolization for hepatocellular carcinoma. A four year study of 127 French patients. Cancer 1994; 74: 16-22.

5 Kasuagi H, Kojima J, Tatsuta M, Okuda S, Sasaki Y, Imaoka $S$, et al. Treatment of hepatocellular carcinoma by transcatheter arterial embolization combined with intraarterial infusion of a mixture of cisplatin and ethiodized iol Gastroenterology 1989; 97: 965-71.

6 Allison D, Jordan JH, Hennesy O. Therapeutic embolisation of the hepatic artery: a review of 75 procedures. tion of the hepatic arter
Lancet 1985 ; i: $595-9$.

7 Madden MV, Krige JEJ, Bailey S, Benningfield SJ, Geddes C, Werner ID, Terblanche J. Randomized trial of targeted chemotherapy with Lipiodol and 5'-epidoxorubicin compared with symptomatic treatment for hepatoma. Gut 1994; 34: 1598-600

8 Farinati F, De Maria N, Fornasiero M, Salvagnini M, Fagiuoli S, Chiarmonte M, et al. Prospective controlled trial with antiestrogen drug tamoxifen in patients with unresectable hepatocellular carcinoma. Dig Dis Sci 1992; 37: 659-62.

9 Elba S, Giannuzzi V, Misciagna G, Manghisi O. Randomized controlled trial of tamoxifen versus placebo in inoperable hepatocellular carcinoma. Italian fournal of in inoperable hepatocellular carcin

Gastroenterology 1994; 26: 66-8.
10 Cerezo FJM, Tomas A, Donoso L, Enriquez J, Guarner C, Balzano J, et al. Controlled trial of tamoxifen in patients with advanced hepatocellular carcinoma. $\mathcal{F}$ Hepatol 1994, 20: 702-6

11 Livraghi T, Bolondi L, Lazzaroni S, Marin G, Morabito A, Rapaccini GL, et al. Percutaneous ethanol injected in the treatment of hepatocellular carcinoma in cirrhosis. A study of 207 patients. Cancer 1992; 69: 925-9.

12 Chen SC, Hsieh MY, Chuang WL, Wang LY, Chang WY. Development of portal vein invasion and its outcome in hepatocellular carcinoma treated by arterial chemoembolization. $f$ Gastroenterol Hepatol 1994; 9: 1-6.

13 Mondazzi L, Bottelli R, Brambilla G, Rampoldi A, Rezakovic I, Zavaglia C, et al. Transcatheter oily chemoembolization for the treatment of hepatocellular chemoembolization for the treatment of hepatocellular Hepatology 1994; 19: 1115-23.

14 Ngan H, Lai CL, Fan ST, Lai ECS, Yuen WK, Tso WK. Treatment of inoperable hepatocellular carcinoma by transcatheter arterial chemoembolization using an emulsion of cisplatin in iodized oil and gelfoam. Clin Radiol 1993; 47: 315-20.

15 Ikeda K, Kumada H, Saitoshi S, Arase Y, Chayama K. Effect of repeated transcatheter arterial embolization on survival time in patients with hepatocellular carcinoma: an analysis by the Cox proportional hazard model. Cancer 1991; 15: 2150-4.

16 Pelletier G, Roche A, Ink O, Anciaux ML, Derhy S Rougier $\mathrm{P}$, et al. A randomized trial of hepatic arterial chemoembolization in patients with unresectable hepatocellular carcinoma. F Hepatol 1990; 11: 181-4.

17 Group d'Etude et de Traitement du Carcinoma Hepatocellulaire. A comparison of lipiodol chemoembolization and conservative treatment for unresectable hepatocellular carcinoma. N Engl f Med 1995; 332: 1256-61.

18 Bismuth $\mathrm{H}$, Morino M, Sherlock D, Castaing D, Miglietta C, Cauquil $\mathrm{P}$, et al. Primary treatment of hepatocellular carcinoma by arterial chemoembolization. Am $\exists$ Surg 1992; 163: 387-94.

19 Yoshimi F, Nagao T, Inoue S, Kawano N, Muto T, Gunji $\mathrm{T}$, et al. Comparison of hepatectomy and transcatheter arterial chemoembolization for the treatment of hepatocellular carcinoma: necessity for prospective randomized trial. Hepatology 1992; 16: 702-6.

20 Yu Y-Q, Xu D-B, Zhou X-D, Lu J-Z, Tang Z-Y, Mack P. Experience with liver resection after hepatic arterial chemoembolization for hepatocellular carcinoma. Cancer 1993; 71: 62-5. 